\title{
Focused ion beams in future nanoscale probe recording
}

\author{
Dmitri Litvinov $^{1}$ and Sakhrat Khizroev ${ }^{2}$ \\ ${ }^{1}$ Applied Physics, University of Michigan, Ann Arbor, MI, USA \\ ${ }^{2}$ Electrical and Computer Engineering, Carnegie Mellon University, Pittsburgh, PA, USA
}

Received 11 January 2002, in final form 19 February 2002

Published 14 March 2002

Online at stacks.iop.org/Nano/13/179

\begin{abstract}
Focused ion beam (FIB) applications in the field of nanoscale probe recording are explored. A detailed description of how to use FIBs for trimming longitudinal and perpendicular magnetic recording and playback devices to dimensions of less than $100 \mathrm{~nm}$ is presented. An experiment was conducted to demonstrate that the magnetoresistive property of a read element, e.g. a giant-magnetoresistive sensor, strongly depends on the focused ion current and the beam accelerating voltage.
\end{abstract}

\section{Introduction}

Despite a history of decades of gloomy predictions of the "nearfuture decline' of the rapid growth of the areal bit density, its progress in the magnetic recording industry continues at a rate of approximately $100 \%$ density increase per year $[1,2]$. The most recent demonstrations of areal densities of approximately $100 \mathrm{Gbit}^{-2}$ bring the information bit sizes to dimensions smaller than $100 \mathrm{~nm} \mathrm{[3].} \mathrm{This} \mathrm{is} \mathrm{approximately} \mathrm{at} \mathrm{the} \mathrm{limit}$ of the capability to define microscopically small devices using cutting-edge optical lithography processing. Hence, today, in the data storage industry there is a strong need to explore processing technologies capable of bringing dimensions of recording devices into the nanoscale range. The most obvious candidates are electron beam and focused ion beam (FIB) technologies [4-7]. It is commonly believed that these new technologies are necessary for extending magnetic recording to areal densities beyond the $1 \mathrm{Tbit}^{-2}$ mark, which, in other words, implies the fabrication of recording devices with dimensions smaller than $10 \mathrm{~nm}$. The electron beam technology has been more extensively explored in the semiconductor industry, because of its more traditional approach to defining microscopically small dimensions via non-direct patterning masks- thus to some degree recalling optical lithography $[4,5]$. Nevertheless, though FIB technology is more different from the traditional optical lithography, its ability as regards direct etching without using extra patterning steps has the potential of emerging as a reliable processing technique, which requires fewer steps than lithography-based processing $[6,7]$. Therefore, the future potential of FIB processing should not be disregarded. The intention of this paper is to summarize the key findings from the authors' experience acquired during years of research on using FIBs to define ultrasmall magnetic recording devices.

\section{Recording magnetic devices}

Current magnetic recording technology is based on using a ring-type magnetic device to record on a medium with an inplane (longitudinally) oriented magnetization [8]. The ringtype magnetic device, called a ring head $(\mathrm{RH})$, is made of a soft-magnetic material, such as permalloy, $\mathrm{Fe} / \mathrm{Co}$ or a $\mathrm{Ni} / \mathrm{Fe}$ compound, using thin-film processing [9]. A schematic diagram of a typical longitudinal recording system is shown in figure 1(a). A separate playback magnetic device, which is located behind the RH, has several possible configurations and is discussed below in more detail [10]. In longitudinal recording, the actual recording is produced by the field emanating from the physical gap of the RH $[7,11]$. Consequently, the trackpitch of a bit pattern written with the RH is defined by the width of the gap region. For example, at $100 \mathrm{Gbit}^{-2}$ areal density, assuming a 4:1 bit cell aspect ratio (BAR), the track-pitch should be approximately $160 \mathrm{~nm}$. If a FIB were used to produce a RH suited to this density, the smallest and thus the most critical feature of the head to be defined via FIB trimming, i.e. the gap width, would have to be smaller than $160 \mathrm{~nm}$.

Because the main goal of this research is to develop a FIB processing technology suitable for future magnetic recording, one should, also, seriously explore so-called perpendicular magnetic recording $[12,13]$. It is believed that perpendicular recording might replace conventional longitudinal recording because, as predicted, the latest areal demonstrations of about $100 \mathrm{Gbit}^{-2} \mathrm{n}^{-2}$ areal density already indicated signs of thermal instabilities in recording media [14]. The most widely used version of the perpendicular recording technology utilizes a single-pole head (SPH) to record on a double-layer medium, which includes a hard layer with a magnetization in the 

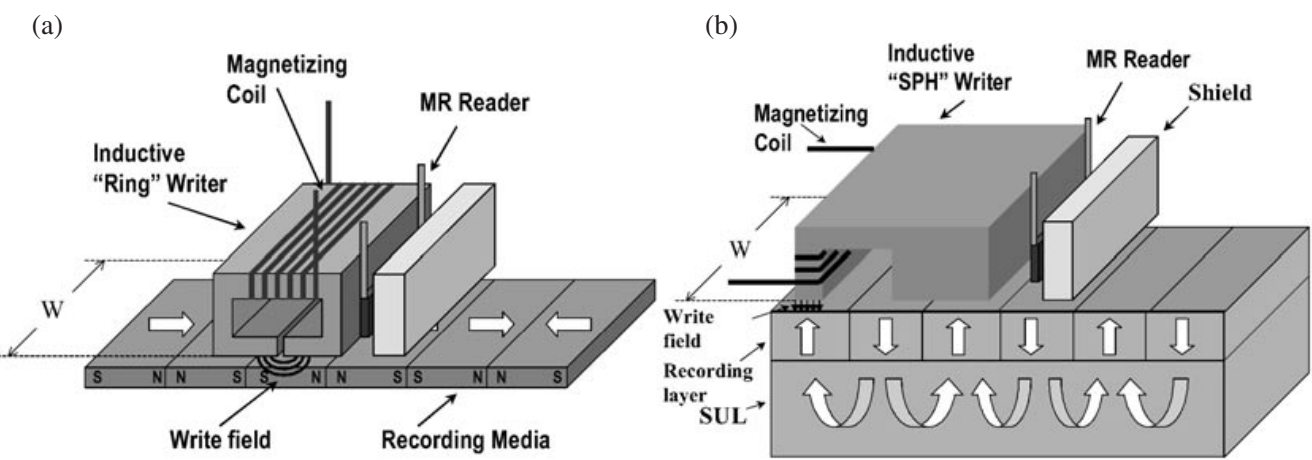

Figure 1. A schematic diagram of typical (a) longitudinal and (b) perpendicular systems.

direction perpendicular to the disk and a soft underlayer (SUL), as shown in figure $1(b)$ [15]. The SPH is different from the $\mathrm{RH}$ in having a significantly larger physical gap thickness. The purpose of the large gap is to make the magnetic flux flow through the SUL, thus increasing the perpendicular component of the recording field $[15,16]$. The use of the SUL allows an approximately twofold increase of the recording field. Unlike in longitudinal recording, the actual recording is produced by the recording field near the trailing edge of the trailing pole of the SPH $[2,17]$. Therefore, definition of the trailing edge of the trailing pole is, also, a critical part of the FIB trimming process.

For a playback head, typically, the same head configuration as for longitudinal recording is utilized. As regards FIB trimming of the playback head, special care should be taken not to destroy the magnetoresistive property of the read element during the FIB etching [18]. Below, a description of the FIB processing of the playback head, as well as of the two write head configurations, RH and SPH, is presented.

\section{FIB trimming set-up}

Regular longitudinal magnetic RHs, made of a relatively highmoment material, $\mathrm{Ni} 45 / \mathrm{Fe} 55$ with a $4 \pi M_{s}$ of approximately $15 \mathrm{kG}$ with a $200 \mathrm{~nm}$ gap, were FIB trimmed from the air-bearing surface (ABS) to define ultranarrow-track-width longitudinal $(\mathrm{RH})$ and perpendicular $(\mathrm{SPH})$ heads [7]. An ultranarrow magnetoresistive reader was defined via FIB trimming a conventional shielded GMR head from the ABS [19].

The FIB trimming was performed using a $5 \mathrm{~nm}$ beam column system, Micrion 2500. During trimming at room temperature and a chamber pressure of approximately $5 \times$ $10^{-8}$ Torr, the gallium-ion beam current was varied, depending on the particular task, from approximately $800 \mathrm{pA}$ down to $1 \mathrm{pA}$ [20]. The stronger the ion current is, the faster the etch rate is. However, also, the stronger the ion current is, the worse the trimming resolution is, as shown in figure 2 . Therefore, there is a trade-off between the etch rate and the trimming resolution. As an example, at a $100 \mathrm{pA}$ ion current it takes approximately 5 min to make a $1 \mu \mathrm{m}$ deep trench with a $5 \mu \mathrm{m} \times 5 \mu \mathrm{m}$ square cross section in a permalloy thin film.

In this paper, the trimmed pole track-width is expected to be of the order of $100 \mathrm{~nm}$, so a beam spot size of approximately $10 \mathrm{~nm}$ is required to provide sufficient resolution. For a

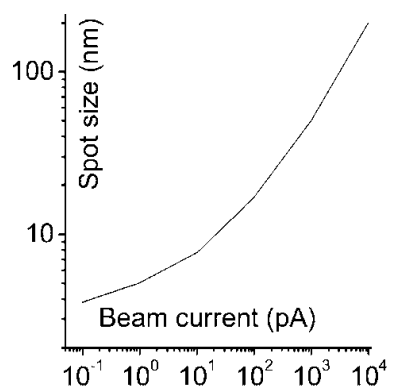

Figure 2. The FIB spot size versus the ion current.

beam spot size of approximately $10 \mathrm{~nm}$, an ion current of approximately $10 \mathrm{pA}$ is sufficient, as seen from figure 2 . However, if so small an ion current were used for etching the entire required region, FIB trimming of one recording head to a track-width of $100 \mathrm{~nm}$ would have taken almost $5 \mathrm{~h}$. Hence, to make the trimming process faster, trimming of each head was performed at a sequence of different ion current values. The simplest scenario consists of two steps. First, relatively large regions with relatively large resolution margins (with a separation between the two $1 \mu \mathrm{m}$ deep trenches of the order of $500 \mathrm{~nm}$ or more) can be trimmed at a relatively large ion current, e.g. 150 pA. Finally, sharp edges can be more precisely defined by trimming small regions near the edges at a relatively small ion current, e.g. $10 \mathrm{pA}$. This two-step trimming process, as shown in figures $3(a)$ and $(b)$, reduces the trimming time per head to approximately a few minutes. Although this time can be much further reduced, into the range of a second per head, by increasing the ion current during the first step, a relatively small current of $150 \mathrm{pA}$ was arbitrarily chosen purely for demonstration purposes. The Micrion 2500 system provides different options for trimming: for example, singlescan and multiple-scan modes. The first and second steps of the two-step process are performed in the multiple-scan (with approximately 1000 scans per trench) and single-scan modes, respectively.

To facilitate clear understanding of the reason for using the different scan types, a basic description of how FIBs can be manipulated to define different topologies of trimmed areas is presented. First of all, there are two major types of trimming scan: single-scan and multiple-scan types. During a singlescan-type trim, the energy dose (proportional to the amount of net charge emitted by the ion source) is distributed uniformly 


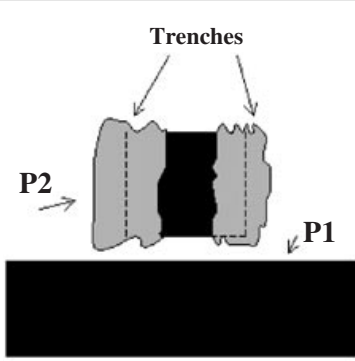

(a)

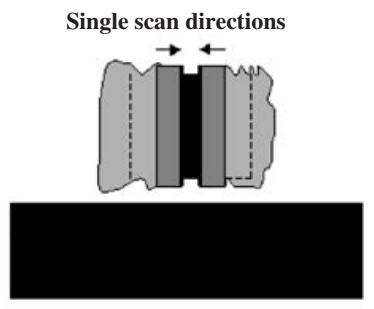

(b)

Figure 3. Diagrams showing: $(a)$ two relatively large trenches with a $500 \mathrm{~nm}$ separation etched at a $150 \mathrm{pA}$ ion current in the multiple-scan mode; and $(b)$ two narrow trenches near the important sides (defining the track-width) with a $100 \mathrm{~nm}$ separation and etched at a $10 \mathrm{pA}$ ion current in the single-scan mode. The arrows show the scan directions.

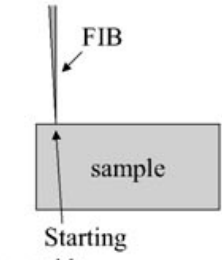

(a) side

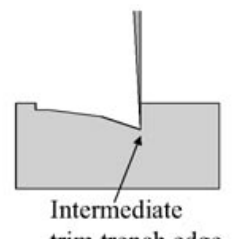

trim trench edge

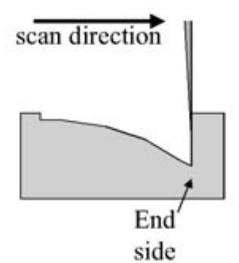

After Nth scan

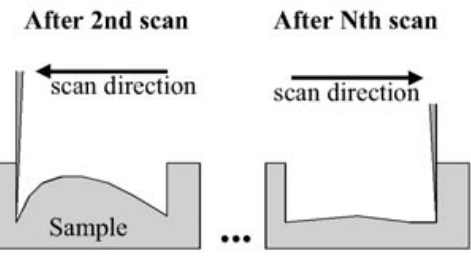

(b)

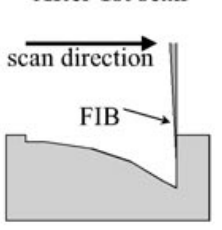

Figure 4. A time sequence for the interaction of the FIB with the sample surface during trimming in the $(a)$ single-scan and (b) multiple-scan modes.

over time so that a desired trim depth is achieved on average for a trench of a desired cross section during only one scan. Below, it is explained why such a charge distribution, uniform in time, does not provide a horizontally flat bottom surface of the trimmed trench during a single scan. The shape of the trench surface during a single-scan etch depends on which side of the trench the trimming was started. The cause of this scan direction dependence is the feature that at each instant of the ion interaction with the sample surface, the environment is different from the environment at an earlier instant. A time sequence for the interaction of the FIB with the sample surface during a single scan is shown in figure $4(a)$. At the starting instant, the entire surface is flat and it does not matter where the first interaction of the FIB with the uniform surface takes place. However, for each of the following interaction events, the effective starting condition is different, because the sample surface is not horizontally flat any longer and therefore it is energetically easier to knock out atoms near the edge part than from the flat part of the trench bottom surface. As a result, the bottom surface is not horizontal after a single-scan trim; rather, it goes deeper to the side of the trench on which the trimming last took place. In contrast, during a trim of the multiple-scan type, the amount of net emitted ion charge is distributed such
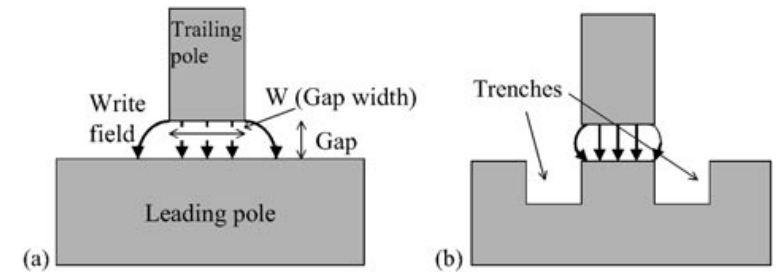

Figure 5. An ABS view of a longitudinal write head (RH) (a) without and $(b)$ with trenches on the leading pole.

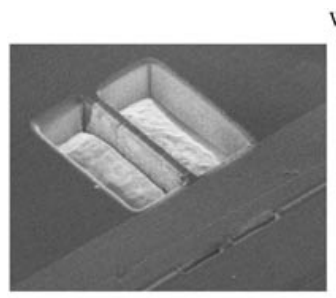

(a)

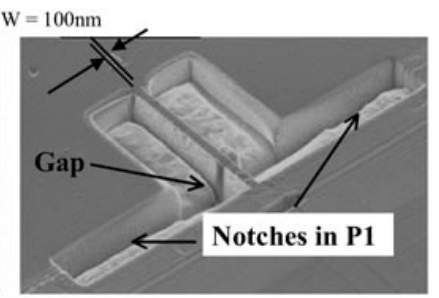

(b)
Figure 6. FIB images of the longitudinal heads $(a)$ without and (b) with notches on the leading pole, P1.

that the FIB scans the surface alternatively back and forth a number of times, as shown in the diagram in figure $4(b)$. In other words, during a trim of the multiple-scan type, due to the FIB alternately travelling in two directions (forward and back) many times, an averaging of the bottom surface with respect to the horizontal level takes place. The larger the number of scans is, the more horizontal the bottom surface is.

Going back to the example of the two-step trimming of a relatively large trench, it is now clear why, besides using a sufficiently small ion current of approximately $10 \mathrm{pA}$ or less during the second step, it also helps at this stage to use the single-scan mode to define a required small track-width as precisely as possible. The most critical trench sides are the sides which define the track-width and, therefore, they are the sides which demand the best possible trimming resolution. Similarly, it should be mentioned that, although the etch depth becomes relatively uniform after multiple-scan trimming used as the first step, as previously explained, the multiple scan creates poorly defined trench edges, as shown in figure $3(a)$ not only due to a relatively large ion current at this stage but also due to the effect of averaging of the alternating scans.

\section{FIB trimming of a longitudinal writing magnetic device}

As previously mentioned, in conventional longitudinal recording, the recorded track-pitch is defined by the trackwidth of the gap region of the RH, as shown in figure 5(a), and thus the track-width is the most critical dimension to be defined by the FIB trimming. However, this rule is not valid when the track-width is comparable to the gap thickness, which is the case in this experiment, in which the track-width and the gap thickness are 100 and $200 \mathrm{~nm}$, respectively. In this case, the contribution of the side (off-track) fields cannot be neglected. Defining side notches in the leading pole, as shown in figure $5(b)$, somewhat reduces the side-field contribution. Therefore, recently, a correspondingly modified longitudinal 

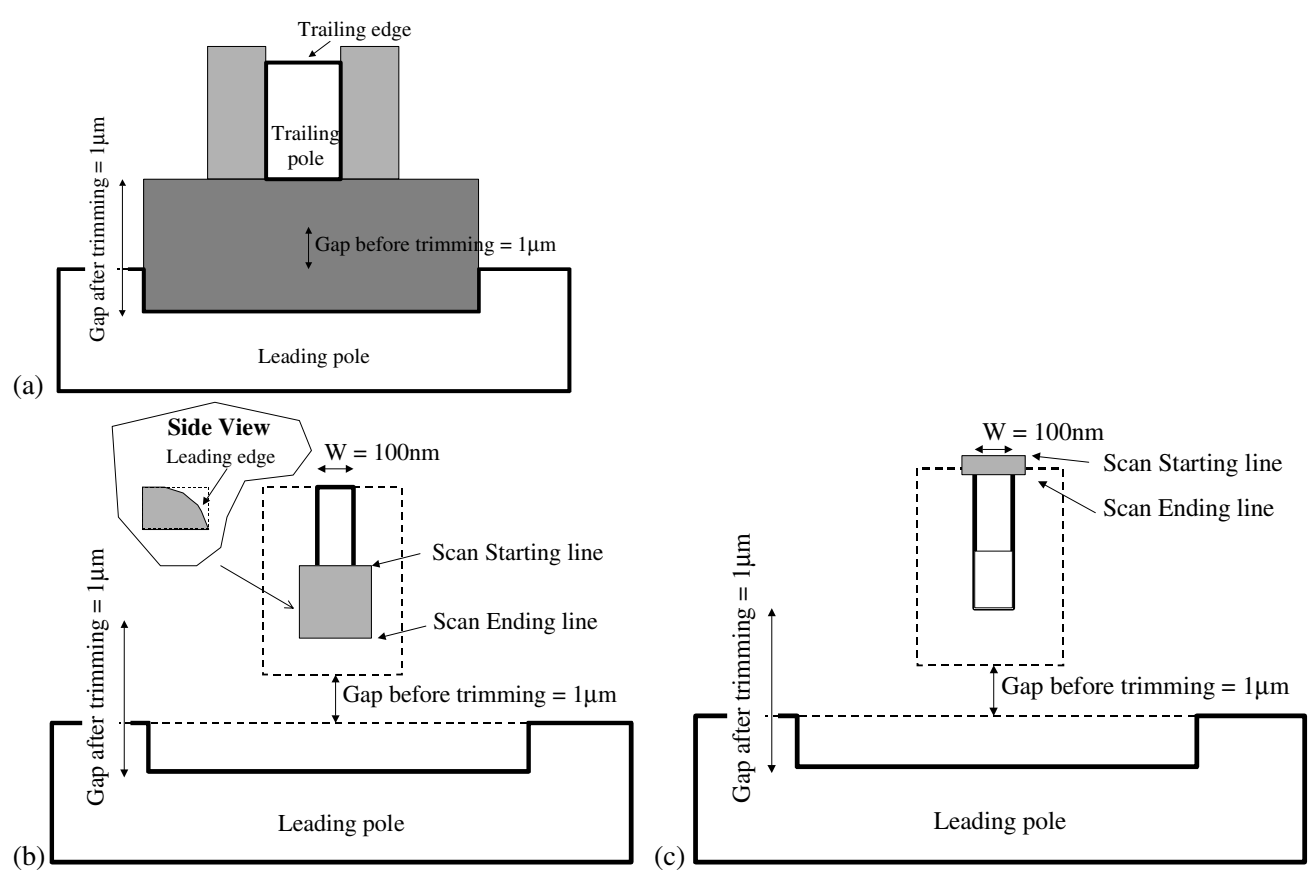

Figure 7. An ABS view showing (a) FIB trimming to define the large gap thickness and the definition of the track-width as a result of a multiple-scan trimming, $(b)$ FIB trimming in the single-scan mode to round off the leading edge, and $(c)$ FIB trimming in the single-scan mode to sharpen the trailing edge. Shadowed regions indicate areas treated by FIB.

writer (with notches in the leading edge) also became a popular design [7]. FIB images of both designs, without and with notches in the leading pole, are shown in figures $6(a)$ and $(b)$, respectively. The exact trimming parameters were found with the help of magnetic field calculations made by the 3D boundary element method (BEM), as explained elsewhere. The main technique used was explained during the description of the example in section 3 and figures $3(a)$ and $(b)$.

\section{FIB trimming of a perpendicular writing magnetic device}

As previously mentioned, a perpendicular $\mathrm{SPH}$, as well as a longitudinal $\mathrm{RH}$, was made by means of FIB trimming of a normal longitudinal RH with a $200 \mathrm{~nm}$ gap thickness. To help with the understanding of the concept of the FIB trimming of a SPH, diagrams corresponding to different steps of the FIB trimming are shown in figures $7(a)-(c)$. Unlike during trimming of a longitudinal RH, the gap thickness was increased via the FIB etching up to approximately $1 \mu \mathrm{m}$ to allow the magnetic flux flow through the SUL, thus enhancing the perpendicular component of the recording field, as described above.

The FIB etch of the gap was performed at a $150 \mathrm{pA}$ current at a $7 \mathrm{C}$ dose in the multiple-scan mode with 1000 scans. Similarly, FIB trimming of the sides was performed under the same conditions, with the track after the side trimming at this stage still remaining wider than the final-aim track-width. As described above, the final track-width is defined via trimming at a $10 \mathrm{pA}$ ion current at a $1 \mathrm{C}$ dose in the single-scan mode. As known from previous modelling results, it makes a more robust ultrahigh-density recording head if the leading edge of the trailing pole is rounded, because the lack of a sharp leading corner guarantees that the field at the leading edge is smaller than the field at the trailing edge and, as a result, the recording is produced by the trailing field and not by the leading field. The required rounding of the leading pole is performed via applying a FIB at a $20 \mathrm{pA}$ ion current and a dose of $0.1 \mathrm{C}$ in the single-scan mode with the starting and the ending scan lines inside the ABS areas of the trailing pole and the gap, respectively, as shown in figure $7(b)$. Also, to enhance the trailing field and the field gradient, extra sharpening of the trailing edge of the trailing pole is required. The sharpening was accomplished via applying a FIB at a $10 \mathrm{pA}$ ion current and a dose of $1 \mathrm{C}$ in the single-scan trim mode with the starting and ending scan lines outside and in the ABS area of the trailing edge of the trailing pole, respectively, as shown in figure $7(c)$. This particular sequence of FIB processing was chosen so that the most critical part of the trimming, which is the trimming of the trailing edge of the trailing pole, is performed as the last step to avoid interaction of the trailing edge with the FIB as much as possible.

A FIB image of a trimmed perpendicular head with a $100 \mathrm{~nm}$ track-width is shown in figure 8 .

A detailed study of the magnetic performance of the FIBtrimmed longitudinal and perpendicular recording heads has been previously reported elsewhere $[6,20]$.

\section{FIB trimming of a playback magnetic device}

As an example of FIB trimming of a playback magnetic device, a normal shielded GMR reader is considered. As previously mentioned, although not optimal, this playback head configuration is still the most widely used even in perpendicular recording. Probably the most critical concern regarding 


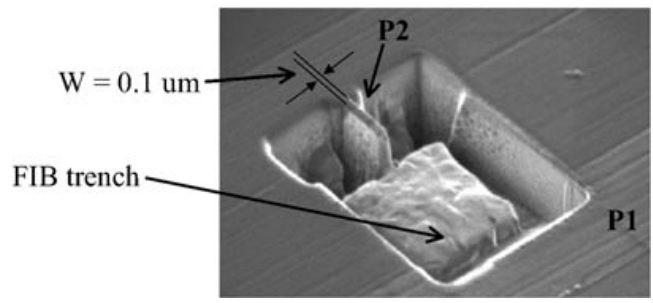

Figure 8. A FIB image of a trimmed perpendicular head.
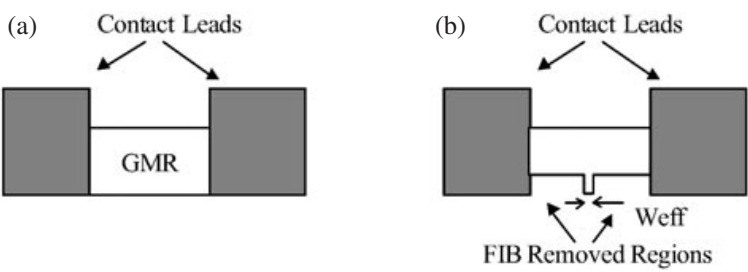

Figure 9. A schematic diagram of a side view of $(a)$ a normal MR head and $(b)$ a trimmed MR head.

FIB trimming of GMR heads is the risk of damaging the magnetoresistive sensitivity of the GMR element during the ion bombardment. Indeed, our experiments show that at a typical ion extraction energy of approximately $50 \mathrm{keV}$, the trimmed GMR heads would stop functioning even at a relatively small ion current value of approximately $10 \mathrm{pA}$. It is known that if subjected to an external energy variation of approximately one order of magnitude larger than $k T$, where $T$ is temperature and $k$ is Boltzmann's constant, the magnetoresistive sensitivity of a typical GMR element can be destroyed.

Also, the magnetic softness and reversibility of softmagnetic materials, of which a recording head is made, are more important in the case of the playback process than in the case of the writing process $[21,22]$. Unlike the writing process, which occurs in the saturation regime, the playback process takes place in a linear magnetic regime and, moreover, the playback signal is proportional to the relative permeability of the head material and is less noisy if the pole material magnetization loop is hysteresis free. The magnetic softness is also sensitive to the interaction of the FIB with the sample. To avoid the destructive influence of the FIB on both the magnetoresistive sensitivity and the magnetic linearity of a sample, trimming was performed at a current of less than $10 \mathrm{pA}$ and an ion extraction energy of less than $20 \mathrm{keV}$.

Schematic diagrams showing side-view cross sections of normal and trimmed GMR heads are shown in figures $9(a)$ and $(b)$, respectively. The trimming can be performed at the wafer level or on a finished head at the ABS level. Although, in the current experiments, FIB trimming was performed at the ABS level, it should be noticed that to improve the GMR fabrication yield, it might be preferable to use trimming at the wafer level because trimming of the thinner layer at the wafer level is easier to control, and also a proper grounding to eliminate the common problem of static discharge can also be more fully accomplished at the wafer level.

For a clear illustration of the functioning of an ultranarrow GMR head after FIB trimming, the following experiment was performed. Both a reader and a writer were trimmed to approximately $100 \mathrm{~nm}$, as shown in a FIB image in figure 10 .

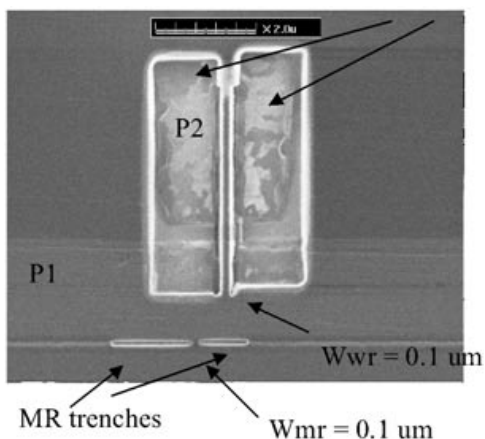

Figure 10. An ABS view of a FIB image of the ABS of a recording head in which both read and write elements are trimmed to a $100 \mathrm{~nm}$ track-width, with an offset of $300 \mathrm{~nm}$ between the centres of the two heads.

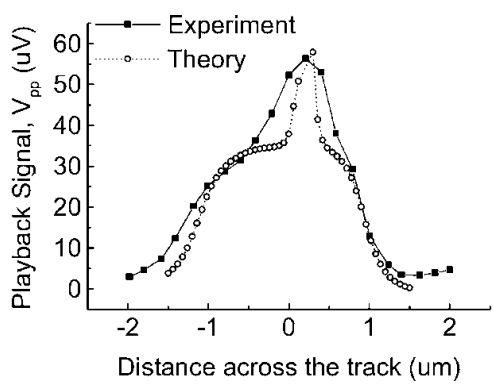

Figure 11. A track profile taken with a trimmed $100 \mathrm{~nm}$ wide MR element at a $50 \mathrm{~nm}$ flying height. The dotted curve represents a theoretical curve.

The etch depths for the GMR element and the writer were $200 \mathrm{~nm}$ and $1 \mu \mathrm{m}$, respectively. In order to confirm that the portion of the MR element that remained at the ABS after trimming is magnetically active, a physical offset of approximately $300 \mathrm{~nm}$ between the centres of the original GMR element and the same GMR element after trimming was defined. A track profile taken by the trimmed $100 \mathrm{~nm}$ wide MR element at a $50 \mathrm{~nm}$ flying height is shown in figure 11. The offset of approximately $300 \mathrm{~nm}$ in the location of the playback maximum corresponds to the physical location of the unetched portion of the trimmed GMR element. The dotted curve in figure 11 corresponds to a theoretical curve calculated using the reciprocity principle [13]. In the calculations, it was assumed that the pole tip material was magnetically soft. The good agreement shows that the MR element is functioning as expected, and that the $100 \mathrm{~nm}$ wide write pole tip has written a well defined track, although the track edge effects in the written track certainly contribute to an additional increase of the playback track-width.

\section{Conclusions}

Applications of FIB technology in the area of magnetic recording were explored. To demonstrate that the FIB technology has the potential to bring the magnetic device dimensions into the nanoscale range, actual write and playback magnetic heads were fabricated using a newly developed FIB process. A method was developed for using a FIB for trimming the write and read heads so that magnetic recording can be extended to densities beyond $1 \mathrm{Tbit}^{-2}$. Write heads of the 
two major types, the longitudinal RH and perpendicular SPH, and a typical shielded GMR head were FIB trimmed down to track-widths smaller than $100 \mathrm{~nm}$. A special experiment was developed to prove the magnetic functionality of the trimmed recording heads.

\section{References}

[1] Khizroev S, Kryder M and Litvinov D 2001 Next generation perpendicular systems IEEE Trans. Magn. 371922

[2] Thompson D A and Best J S 1998 The extendibility of magnetic recording for data storage IBM Executive Briefing (January)

[3] Liu F, Stoev K, Leal L, Wang J, Chen Y, Shi S, Tong H, Lederman M and Re M 2001 Perpendicular high areal densities and high data rates AIT-MINT 2001 Workshop on Magnetic Information Storage Technology (March, 2001) presentation

[4] Davari B, Dennard R and Shahidi G G 1995 CMOS scaling for high performance and low power-the next ten years Proc. IEEE 83595

[5] Fontana R E Jr, MacDonald S A, Santini H A A and Tsang C 1998 Process consideration for critical features in high areal density thin film magnetoresistive heads: a review IEEE Trans. Magn. 35806

[6] Khizroev S K, Kryder M H, Ikeda Y, Rubin K, Arnett P, Best $\mathrm{M}$ and Thompson D A 1999 Recording heads with trackwidths suitable for $100 \mathrm{Gbit}^{-2}$ density IEEE Trans. Magn. 352544

[7] Hill A R 1968 Nature 218292

[8] Westmijze W K 1953 Studies on magnetic recording Philips Res. Rep. 8148

Westmijze W K 1953 Studies on magnetic recording Philips Res. Rep. 8161

Westmijze W K 1953 Studies on magnetic recording Philips Res. Rep. 8245

Westmijze W K 1953 Studies on magnetic recording Philips Res. Rep. 8343
[9] Jones R E Jr 1998 Fabrication of film heads with high moment materials Acta Mater. 463805

[10] O'Connor D J, Shelledy F B and Heim D E 1985 Mathematical model of a magnetoresistive read head for a magnetic tape drive IEEE Trans. Magn. 211560

[11] Khizroev S, Chomko R, Chen G, Liu Y, Mountfield K, Kryder M and Litvinov D 2000 Perpendicular systems above 100 Gbit in $^{-2}$ density Intermag 2000 (April, 2000) presentation CB-07

[12] Iwasaki S and Nakamura Y 1977 An analysis for the magnetization mode for high density magnetic recording IEEE Trans. Magn. 131272

[13] Khizroev S, Bain J A and Kryder M H 1997 Considerations in the design of probe heads for $100 \mathrm{Gbit}^{2}$ recording density IEEE Trans. Magn. 332893

[14] Charap S H 1997 Thermal stability of recorded information at high densities IEEE Trans. Magn. 33978

[15] Litvinov D, Kryder M and Khizroev S 2001 Recording physics of perpendicular media: soft underlayer J. Magn. Magn. Mater. 23284

[16] Litvinov D, Chomko R, Chen G, Abelmann L, Ramstock K and Khizroev S 2001 Micromagnetics of a soft underlayer IEEE Trans. Magn. 362483

[17] Khizroev S, Liu Y, Mountfield K, Kryder M and Litvinov D 2002 Physics of perpendicular magnetic recording: writing process J. Magn. Magn. Mater. at press

[18] Dieny B et al 1991 J. Appl. Phys. 694774

[19] Heim D E 1994 Design and operation of spin valve sensors IEEE Trans. Magn. 30316

[20] Khizroev S 1999 Electrical and computer engineering $P h D$ Thesis Carnegie Mellon University, Pittsburgh, PA

[21] Khizroev S, Jayasekara W, Bain J A, Jones R E Jr and Kryder M H 1998 MFM quantification of magnetic fields generated by ultra-small single pole perpendicular heads IEEE Trans. Magn. 342030

[22] Abelmann L, Khizroev S, Litvinov D, Bain J A, Zhu J, Kryder M H, Ramstock K and Lodder C 2000 Micromagnetic simulation of ultra-small single pole perpendicular heads J. Appl. Phys. 876636 